\title{
Calcium blockade - nifedipine in the treatment of hypertrophic cardiomyopathy
}

\author{
Beverly H. Lorell, William Grossman, Walter J. Paulus, William E. Craig and \\ Joseph P. Murgo
}

The Department of Medicine, Harvard Medical School, Boston, Massachusetts, and the Department of Medicine, Brooke Army Medical Center, Fort Sam Houston, San Antonio, Texas, USA.

\begin{abstract}
Summary: The acute effects of the calcium channel blocking agent nifedipine in hypertrophic cardiomyopathy are described. Improved diastolic function was suggested by the increase in the rate of filling of the left ventricle and the fall in left ventricular end-diastolic pressure. Left ventricular relaxation time was shortened. There was a downward shift in the left ventricular pressure-echo dimension relationship, suggesting increased left ventricular distensibility. Nifedipine caused a fall in systolic, systemic blood pressure and an increase in heart rate, but no change in the cardiac index.

The reasons for the improvement in the diastolic function were thought to be systemic vasodilatation and change in left ventricular loading conditions, coronary vasodilatation with reduction of subendocardial left ventricular ischaemia and a direct myocardial effect and modification of myocardial calcium availability.
\end{abstract}

Hypertrophic cardiomyopathy is frequently associated with abnormal left ventricular (LV) relaxation and diastolic filling patterns (Hanrath et al., 1980; Banau et al., 1981). These disturbances of diastolic function are strikingly similar to those which can be induced in the non-hypertrophied LV during demand ischaemia (Bourdillon et al., 1983). In this regard, previous studies in our laboratory in coronary disease patients showed that the acute administration of the calcium blocker nifedipine attenuated the prolongation of LV relaxation and upward shift in the LV pressure-volume curve seen during angina induced by pacing tachycardia (Lorell et al., 1981). For these reasons, we undertook a prospective study of the acute effects of nifedipine on LV relaxation and diastolic filling in 15 patients with hypertrophic cardiomyopathy (HCM) (Lorell et al., 1982).

\section{Haemodynamic studies with nifedipine in hypertrophic cardiomyopathy}

In this study, M-mode echocardiograms and electrocardiograms were recorded at baseline and $20 \mathrm{~min}$ after nifedipine (10 $\mathrm{mg}$ sublingual). In 10 patients, echocardiograms were recorded simultaneously with LV micromanometer tip pressure, systemic arterial

Correspondence and present address: Beverly $\mathbf{H}$. Lorell, M.D., Cardiovascular Division, Harvard Medical School, Department of Medicine at the Beth Israel Hospital, 330 Brookline Avenue, Boston, Massachusetts 02215, USA. pressure, and cardiac output. LV pressures and echo dimensions were digitized and interfaced with a microprocessor to derive the peak rates of LV filling (peak rate of diastolic dimension change) and diastolic posterior wall thinning. The LV isovolumic relaxation time (IVRT) was calculated as the interval between aortic valve closure and mitral valve opening (the normal range \pm s.d. in our laboratory is $60 \pm 7 \mathrm{~ms}$. Nifedipine administration was associated with a decrease in the prolonged IVRT from $112 \pm 26$ to $83 \pm 23 \mathrm{~ms}(P<0.0001)$. Similar improvement was noted in the changes in the peak LV filling rate (normal, $118 \pm 7 \mathrm{~mm} / \mathrm{s}$ ) and peak rate of diastolic posterior wall thinning (normal, $81 \pm 11 \mathrm{~mm} / \mathrm{s}$ ). After nifedipine administration, there was an increase in the depressed rate of diastolic filling from $72 \pm 37$ to $101 \pm 39 \mathrm{~mm} / \mathrm{s}(P<0.01)$, and an increase in the depressed rate of $L V$ posterior wall thinning from $47 \pm 31$ to $68 \pm 36 \mathrm{~mm} / \mathrm{s}(P<0.001)$.

In the 10 patients in whom haemodynamics were measured, nifedipine was associated with a fall in systolic arterial pressure from $124 \pm 20$ to $110 \pm 13 \mathrm{~mm} \mathrm{Hg}(P<0.01)$, and an increase in heart rate from $74 \pm 14$ to $84 \pm 17$ beats $/ \mathrm{min}(P<0.05)$. However, individual responses varied, and analysis showed that an improvement in LV isovolumic relaxation and diastolic filling occurred after nifedipine irrespective of change in blood pressure or heart rate. For the group, nifedipine administration was associated with a fall in systemic vascular resistance and no change in cardiac index $(\mathrm{CI})$ or extent of systolic shortening. After nifedipine administration, 
one of 8 patients without a resting LV outflow gradient developed a gradient, while 2 patients experienced a fall in resting gradient.

Diastolic haemodynamics also improved after nifedipine. LV end-diastolic pressure (LVEDP) fell in $7 / 9$ patients in whom it was elevated at baseline. For the group, LVEDP fell from $22 \pm 8$ to $18 \pm 6 \mathrm{~mm} \mathrm{Hg}$ $(P<0.01)$. These changes were associated with an improvement in the prolonged $L V$ relaxation time constant (tau) which shortened from $63 \pm 20$ to $49 \pm 11 \mathrm{~ms}(P<0.05)$. Analysis of the relationship between LV pressure and echocardiographic dimension suggested that the fall in LVEDP following nifedipine administration was related to a downward shift in the LV pressure-dimension relation, suggesting improved LV distensibility.

Of interest, comparable findings have been reported by Suwa et al (1983) who studied the haemodynamic effects of nifedipine administration in 13 patients with HCM and in 8 normal volunteers. In that study, an increase in heart rate and reduction in LV afterload were observed after nifedipine in both HCM patients and normals. In HCM patients, the prolonged LV isovolumic relaxation time in HCM was normalized $(93 \pm 21$ to $69 \pm 19 \mathrm{~ms}, P<0.001)$ after nifedipine administration, with improvement in the prolonged $L V$ peak filling rate $(-4.7 \pm 2.0$ to $-5.8 \pm 2.4 \mathrm{circ} / \mathrm{s}$, $P<0.001)$ and shortening of the prolonged time to peak filling rate $(120 \pm 26$ to $107 \pm 20 \mathrm{~ms}, P<0.05)$. In contrast, these diastolic parameters of relaxation and filling were not significantly changed in normals after nifedipine.

These observations suggest that the acute administration of the calcium blocker nifedipine is associated with improved LV diastolic function in HCM patients, and that the effects of calcium blockers on diastolic function may differ in patients with hypertrophied and non-hypertrophied normal left ventricles. The mechanisms which contribute to the improvement of diastolic function in patients with HCM after calcium blocker administration are now shown. Some possible mechanisms include: (1) systemic vasodilatation and alteration of LV loading conditions; (2) coronary vasodilatation and reduction of subendocardial ischaemia of the hypertrophied LV; (3) a direct myocardial effect and modification of myocardial calcium availability.

\section{Altered LV loading or muscle inactivation?}

To evaluate the relative importance of altered $\mathrm{LV}$ loading versus improved muscle inactivation, we prospectively investigated the effects of nifedipine in 10 patients with non-obstructive HCM (Paulus et al., 1983). To distinguish between these mechanisms, we compared the effects of nifedipine and the vasodilator nitroprusside on LV diastolic properties using simultaneous LV micromanometer pressure and echocardiographic measurements. Both drugs were associated with the same reduction in LV systolic load, since LV systolic pressure was comparable during nitroprusside infusion $(132 \pm 38 \mathrm{~mm} \mathrm{Hg})$ and after nifedipine $(132 \pm 32 \mathrm{~mm} \mathrm{Hg})$, as was $\mathrm{LV}$ end-systolic dimension (Nitroprusside 27.9 \pm 11.3 , nifedipine $28.0 \pm 10.4 \mathrm{~mm}$ ). Further, heart rate was comparable during nitroprusside and nifedipine administration. Both drugs were associated with an improvement in diastolic relaxation and filling parameters relative to baseline. However, compared with nitroprusside, nifedipine was associated with significantly greater improvement in the $\mathrm{LV}$ isovolumic relaxation time (nitroprusside $95 \pm 26$, nifedipine $84 \pm 25 \mathrm{~ms}, P<0.05$ ) and peak rates of LV filling (nitroprusside $89 \pm 46$, nifedipine $106 \pm 50 \mathrm{~mm} / \mathrm{s}, P<0.05)$ and diastolic posterior wall thinning (nitroprusside $64 \pm 41$, nifedipine $90 \pm 54 \mathrm{~mm} / \mathrm{s}, P<0.05)$.

During nitroprusside treatment, the fall in LVEDP $(22 \pm 11$ to $17 \pm 11 \mathrm{~mm} \mathrm{Hg}, P<0.05)$ was related to a decrease in $\mathrm{LV}$ end-diastolic dimension, indicative of a reduction in the extent of LV diastolic filling. However, the decrease in LVDEP after nifedipine $(22 \pm 11$ to $18 \pm 10 \mathrm{~mm} \mathrm{Hg}, \quad P<0.05)$ was not associated with a fall in LV end-diastolic dimension, suggestive of an increase in LV distensibility.

\section{Comment}

These observations indicate that LV systolic unloading is not the only mechanism responsible for the improvement in diastolic function in HCM after calcium blocker administration. The improvement in diastolic function appears to result not only from systemic vasodilatation but also from enhanced cardiac muscle inactivation. The relative contributions of subendocardial ischaemia and/or altered intracellular $\mathrm{Ca}^{++}$regulation to abnormal cardiac muscle inactivation in HCM are as yet poorly understood. Calcium channel blockers, including nifedipine, possess coronary vasodilating properties (Malakoff et al., 1982). In the hypertrophied $L V$, coronary vasodilatation at the level of the microvasculature might modify subendocardial ischaemia (Pasternac et al., 1982; Bache et $a l ., 1984$ ) and attenuate ischaemia-induced changes in LV diastolic relaxation and filling (Bourdillon et al., 1983; Serizawa et al., 1981).

In regard to the possibility that intracellular calcium regulation is altered, preliminary observations by Morgan \& Morgan (1985) using the aequorin indicator technique suggest that the intracellular $\mathrm{Ca}^{++}$ transient is aberrant and prolonged in $\mathrm{LV}$ tissue from HCM patients. Further, this aberrant $\mathrm{Ca}^{++}$transient was modified by tissue exposure to the $\mathrm{Ca}^{++}$blocker 
verapamil. In a model of calcium overload, the effects of the calcium blocker verapamil on $\mathrm{Ca}^{++}$overload in cultured heart cells were examined (Lorell \& Barry, 1984). Exposure of cultured heart cells to high extracellular $\mathrm{Ca}^{++}$resulted in prolongation of the rate of diastolic relaxation, failure of the cells to relax to baseline, and reduction in the extent of cell shortening. The addition of verapamil during calcium overload

\section{References}

BACHE, R.J., ARENT ZEN, C.E., SIMON, A.P. \& VROBEL, T.R. (1984). Abnormalities in myocardial perfusion during tachycardia in dogs with left ventricular hypertrophy: metabolic evidence for myocardial ischemia. Circulation, $69,409$.

BONOW, R.O., ROSING, D.R., BACHARACH, S.L., GREEN, M.V., KENT, K.M., LIPSON, L.C., MARON, B.J., LEON, M.B. \& EPSTEIN, S.E. (1981). Effects of verapamil on systolic function and diastolic filling in patients with hypertrophic cardiomyopathy. Circulation, 64, 787.

BOURDILLON, P.D., LORELL, B.H., MIRSKY, I., PAULUS, W.J., WYNNE, J. \& GROSSMAN, W. (1983). Increased regional myocardial stiffness of the left ventricle during pacing-induced angina in man. Circulation, 67, 316.

HANRATH, P., MATHEY, D.G., SIEGERT, R. \& BLEIFELD, W. (1980). Left ventricular relaxation and filling pattern in different forms of left ventricular hypertrophy: an echocardiographic study. American Journal of Cardiology, 45, 15.

LORELL, P.H., TURI, Z. \& GROSSMAN, W. (1981). Modification of left ventricular response to pacing tachycardia by nifedipine in patients with hypertrophic cardiomyopathy. American Journal of Medicine, 667.

LORELL, B.H., PAULUS, W.I., GROSSMAN, W., WYNNE, J. \& COHN, P.F. (1982). Modification of abnormal left ventricular diastolic properties by nifedipine in patients with hypertrophic cardiomyopathy. Circulation, 65, 499.

LORELL, P.H. \& BARRY, W.H. (1984). Effects of verapamil on contraction and relaxation of cultured chick embryo ventricular cells during calcium overload. Journal of the American College of Cardiology, 3, 341. caused a marked improvement in the rate and extent of diastolic relaxation.

These findings again emphasize that the effects of calcium-blockers on diastolic function in patients are likely to differ depending on the presence of normal LV myocardium versus abnormal myocardium due to hypertrophy, ischaemia, or altered $\mathrm{Ca}^{++}$regulation.

MALAKOFF, R.F., LORELL, B.H., MUDGE, G.H., et al. (1982). Beneficial effects of nifedipine on regional myocardial blood flow in patients with coronary artery disease. Circulation, 65 (Suppl 1), 32.

MORGAN, J.P. \& MORGAN, K.G. (1985). Calcium and cardiovascular function: intracellular calcium levels during contraction and relaxation of mammalian cardiac vascular smooth muscle as detected by aequorin. American Journal of Medicine, 77, 33.

PASTERNAC, A., NOBLE. J., STREULENS, Y., ELIE, R., HENSCHKE, C. \& BOURASSA, M.G. (1982). Pathophysiology of chest pain in patients with cardiomyopathies and normal coronaries. Circulation, 65, 778.

PAULUS, W.I., LORELL, B.H., CRAIG, W.E., WYNNE, J.. MURGO, J.P. \& GROSSMAN, W. (1983). Comparison of the effects of nitroprusside and nifedipine on diastolic properties in patients with hypertrophic cardiomyopathy: altered left ventricular loading or improved muscle inactivation? Journal of the American College of Cardiology 2, 879.

SERIZAWA, T., VOGEL, W.M., APSTEIN, C.S. \& GROSSMAN, W. (1981). Comparison of acute alterations in left ventricular relaxation and diastolic chamber stiffness induced by hypoxia and ischemia. Role of oxygen supply demand imbalance. Journal of Clinical Investigation, 68, 91.

SUWA, M., YUZO, H. \& KAWAMURA, K. (1983). Effects of nifedipine on left ventricular systolic and diastolic function in hypertrophic nonobstructive cardiomyopathy: an echocardiographic study. Journal of Cardiovascular Ultrasonography, 2, 9. 